\title{
NON-COMMUTATIVE DISC ALGEBRAS AND THEIR REPRESENTATIONS
}

\author{
GELU POPESCU
}

(Communicated by Palle E. T. Jorgensen)

ABstract. It is shown that the smallest closed subalgebra

$$
\operatorname{Alg}\left(I_{\mathcal{K}}, V_{1}, \ldots, V_{n}\right) \subset \mathcal{B}(\mathcal{K}) \quad(n=2,3, \ldots, \infty)
$$

generated by any sequence $V_{1}, \ldots, V_{n}$ of isometries on a Hilbert space $\mathcal{K}$ such that $V_{1} V_{1}^{*}+\cdots+V_{n} V_{n}^{*} \leq I_{\mathcal{K}}$ is completely isometrically isomorphic to the non-commutative "disc" algebra $\mathcal{A}_{n}$ introduced in Math. Scand. 68 (1991), 292-304. We also prove that for $n \neq m$ the Banach algebras $\mathcal{A}_{n}$ and $\mathcal{A}_{m}$ are not isomorphic. In particular, we give an example of two non-isomorphic Banach algebras which are completely isometrically embedded in each other.

The completely bounded (contractive) representations of the "disc" algebras $\mathcal{A}_{n}(n=2,3, \ldots, \infty)$ on a Hilbert space are characterized. In particular, we prove that a sequence of operators $A_{1}, A_{2}, \ldots$ is simultaneously similar to a contractive sequence $T_{1}, T_{2}, \ldots$ (i.e., $T_{1} T_{1}^{*}+\cdots+T_{n} T_{n}^{*} \leq I$ ) if and only if it is completely polynomially bounded.

The first cohomology group of $\mathcal{A}_{n}$ with coefficients in $\mathbb{C}$ is calculated, showing, in particular, that the disc algebras are not amenable. Similar results are proved for the non-commutative Hardy algebras $F_{n}^{\infty}$ introduced in Math. Scand. 68 (1991), 292-304.

The right joint spectrum of the left creation operators on the full Fock space is also determined.

\section{INTRODUCTION AND PRELIMINARIES}

Let $\mathcal{H}$ be a Hilbert space and $B(\mathcal{H})$ the set of bounded linear operators on $\mathcal{H}$. We identify $M_{m}(B(\mathcal{H}))$, the set of $m \times m$ matrices with entries from $B(\mathcal{H})$, with $B(\underbrace{\mathcal{H} \oplus \cdots \oplus \mathcal{H}}_{\text {m-times }})$. Thus we have a natural $C^{*}$-norm on $M_{m}(B(\mathcal{H}))$. If $X$ is an

operator space, i.e., a closed subspace of $B(\mathcal{H})$, we consider $M_{m}(X)$ as a subspace of $M_{m}(B(\mathcal{H}))$ with the induced norm. Let $X, Y$ be operator spaces and $u: X \rightarrow Y$ a linear map. Define $u_{m}: M_{m}(X) \rightarrow M_{m}(Y)$ by

$$
u_{m}\left[\left(x_{i j}\right)\right]=\left[\left(u\left(x_{i j}\right)\right)\right] .
$$

We say that $u$ is completely bounded ( $c b$ in short) if

$$
\|u\|_{c b}=\sup _{m \geq 1}\left\|u_{m}\right\|<\infty .
$$

Received by the editors January 30, 1995

1991 Mathematics Subject Classification. Primary 47D25; Secondary 47A67.

(C)1996 American Mathematical Society 
If $\|u\|_{c b} \leq 1$ (resp. $\quad u_{m}$ is an isometry for any $m \geq 1$ ), then $u$ is completely contractive (resp. isometric), and if $u_{m}$ is positive for all $m$, then $u$ is called completely positive. In the following we fix $n=1,2,3, \ldots, \infty$.

Let us consider the full Fock space [E]

$$
F^{2}\left(H_{n}\right)=\mathbf{C} 1 \oplus \bigoplus_{m \geq 1} H_{n}^{\otimes m}
$$

where $H_{n}$ is an $n$-dimensional complex Hilbert space with orthonormal basis $\left\{e_{1}, e_{2}, \ldots, e_{n}\right\}$ if $n$ is finite, or $\left\{e_{1}, e_{2}, \ldots\right\}$ if $n=\infty$. For each $i=1,2, \ldots$, $S_{i} \in B\left(F^{2}\left(H_{n}\right)\right)$ is the left creation operator with $e_{i}$, i.e., $S_{i} \xi=e_{i} \otimes \xi, \xi \in F^{2}\left(H_{n}\right)$. We shall denote by $\mathcal{P}_{n}$ the set of all $p \in F^{2}\left(H_{n}\right)$ of the form

$$
p=a_{0}+\sum_{\substack{1 \leq i_{1}, \ldots, i_{k} \leq n \\ 1 \leq k \leq m}} a_{i_{1} \cdots i_{k}} e_{i_{1}} \otimes \cdots \otimes e_{i_{k}}, \quad m \in \mathbb{N},
$$

where $a_{0}, a_{i_{1} \ldots i_{k}} \in \mathbf{C}$ and the sum contains only a finite number of summands. The set $\mathcal{P}_{n}$ may be viewed as the algebra of polynomials in $n$ non-commuting indeterminates, with $p \otimes q, p, q \in \mathcal{P}_{n}$, as multiplication. Define $F_{n}^{\infty}$ as the set of all $g \in F^{2}\left(H_{n}\right)$ such that

$$
\|g\|_{\infty}:=\sup \left\{\|g \otimes p\|_{2}: p \in \mathcal{P}_{n},\|p\|_{2} \leq 1\right\}<\infty
$$

where $\|\cdot\|=\|\cdot\|_{F^{2}\left(H_{n}\right)} \cdot\left(F_{n}^{\infty},\|\cdot\|_{\infty}\right)$ is a non-commutative Banach algebra [Po3]. We denote by $\mathcal{A}_{n}$ the closure of $\mathcal{P}_{n}$ in $\left(F_{n}^{\infty},\|\cdot\|_{\infty}\right)$. The Banach algebra $F_{n}^{\infty}$ (resp. $\mathcal{A}_{n}$ ) can be viewed as a non-commutative analogue of the Hardy space $H^{\infty}$ (resp. disc algebra); when $n=1$ they coincide.

Let $\left(B(\mathcal{H})^{n}\right)_{1}$ denote the unit ball of $\left(B(\mathcal{H})^{n}\right)_{1}$, i.e.,

$$
\left(B(\mathcal{H})^{n}\right)_{1}=\left\{\left(T_{1}, \ldots, T_{n}\right) \in B(\mathcal{H})^{n}: \sum_{i=1}^{n} T_{i} T_{i}^{*} \leq I_{\mathcal{H}}\right\} .
$$

For any sequence $T_{1}, T_{2}, \ldots, T_{n} \in B(\mathcal{H})$ and $p \in \mathcal{P}_{n}$ given by (1.1) we denote by $p\left(T_{1}, \ldots, T_{n}\right)$ the operator acting on $\mathcal{H}$, defined by

$$
p\left(T_{1}, \ldots, T_{n}\right)=a_{0} I_{\mathcal{H}}+\sum a_{i_{1} \ldots i_{k}} T_{i_{1}} \cdots T_{i_{k}} .
$$

The von Neumann inequality [vN],[SzF] for $\left(B(\mathcal{H})^{n}\right)_{1}$ (see [Po3]) asserts that if $\left(T_{1}, \ldots, T_{n}\right) \in\left(B(\mathcal{H})^{n}\right)_{1}$ and $p \in \mathcal{P}_{n}$, then

$$
\left\|p\left(T_{1}, \ldots, T_{n}\right)\right\| \leq\left\|p\left(S_{1}, \ldots, S_{n}\right)\right\|=\|p\|_{\infty} .
$$

According to $[\mathrm{Po} 3]$ the mapping

$$
\Psi: \mathcal{A}_{n} \rightarrow B(\mathcal{H}) ; \quad \Psi(f)=f\left(T_{1}, \ldots, T_{n}\right)
$$

is a contractive homomorphism.

A sequence $A_{1}, \ldots, A_{n} \in B(\mathcal{H})$ will be called completely polynomially bounded (in short c.p.b.) if there is a constant $C$ such that for all $m$ and all $m \times m$ matrices $\left(p_{i j}\right)$ with $p_{i j} \in \mathcal{P}_{n}$ we have

$$
\left\|\left(p_{i j}\left(A_{1}, \ldots, A_{n}\right)\right)\right\|_{M_{m}(B(\mathcal{H}))} \leq C\left\|\left(p_{i j}\right)\right\|_{M_{m}\left(\mathcal{A}_{n}\right)} .
$$

Here of course we consider $\mathcal{A}_{n}$ as a subalgebra of the $C^{*}$-algebra $C^{*}\left(S_{1}, \ldots, S_{n}\right)$ (see $[\mathrm{Po} 3])$. Notice that $A_{1}, \ldots, A_{n}$ is c.p.b. if and only if the homomorphism $p \rightarrow$ $p\left(A_{1}, \ldots, A_{n}\right)$ defines a completely bounded homomorphism from the "disc" algebra $\mathcal{A}_{n}$ into $B(\mathcal{H})$. All the above considerations hold true for $n=\infty$ in a slightly adapted version (see also [Po3]). 


\section{Completely bounded Representations of $\mathcal{A}_{n}$}

It is well known that an operator $T \in B(\mathcal{H})$ is a contraction if and only if it gives rise to a completely contractive representation of the classical disc algebra. In what follows we get an extension of this result to our non-commutative setting.

Theorem 2.1. Let $A_{1}, \ldots, A_{n}$ be in $B(\mathcal{H})$. Then $\left[A_{1}, \ldots, A_{n}\right]$ is a contraction if and only if the map

$$
\Phi: \mathcal{P}_{n} \rightarrow B(\mathcal{H}) ; \quad \Phi(p)=p\left(A_{1}, \ldots, A_{n}\right)
$$

is completely contractive.

Proof. Assume that $\left[A_{1}, \ldots, A_{n}\right]$ is a contraction. According to [Po2] there is a sequence $\left\{V_{1}, \ldots, V_{n}\right\}$ of isometries on a Hilbert space $\mathcal{K} \supset \mathcal{H}$ such that

$$
\sum_{i=1}^{n} V_{i} V_{i}^{*} \leq I_{\mathcal{K}} \text { and }\left.V_{i}^{*}\right|_{\mathcal{H}}=A_{i}^{*}, i=1,2, \ldots, n .
$$

Using the result from [Po5], the map $\Psi: \mathcal{P}_{n} \rightarrow C^{*}\left(V_{1}, \ldots, V_{n}\right)$ defined by

$$
\Psi(p)=p\left(V_{1}, \ldots, V_{n}\right)
$$

can be extended to a $*$-representation of $C^{*}\left(S_{1}, \ldots, S_{n}\right)$ by setting

$$
\Phi\left(S_{i_{1}} \cdots S_{i_{k}} S_{j_{1}}^{*} \cdots S_{j_{p}}^{*}\right)=V_{i_{1}} \cdots V_{i_{k}} V_{j_{1}}^{*} \cdots V_{j_{p}}^{*},
$$

where $1 \leq i_{1}, \ldots, i_{k}, j_{1}, \ldots, j_{p} \leq n$, and by extending it by linearity. In particular we have $\|\Psi\|_{c b} \leq 1$. According to (2.1) we have

$$
\Phi(p)=p\left(A_{1}, \ldots, A_{n}\right)=\left.P_{\mathcal{H}} p\left(V_{1}, \ldots, V_{n}\right)\right|_{\mathcal{H}},
$$

which together with (2.2) implies

$$
\Phi(f)=f\left(A_{1}, \ldots, A_{n}\right)=\left.P_{\mathcal{H}} f\left(V_{1}, \ldots, V_{n}\right)\right|_{\mathcal{H}}=\left.P_{\mathcal{H}} \Psi(f)\right|_{\mathcal{H}},
$$

for all $f \in \mathcal{A}_{n}$. Therefore $\|\Phi\|_{c b} \leq\|\Psi\|_{c b} \leq 1$.

Conversely, suppose that $A_{1}, \ldots, A_{n} \in B(\mathcal{H})$ such that the map

$$
\Phi: \mathcal{P}_{n} \rightarrow B(\mathcal{H}) ; \quad \Phi(p)=p\left(A_{1}, \ldots, A_{n}\right)
$$

is completely contractive. In particular we have

$$
\left\|\left[\begin{array}{cccc}
A_{1} & A_{2} & \cdots & A_{n} \\
0 & 0 & \cdots & 0 \\
\vdots & \vdots & & \vdots \\
0 & 0 & \cdots & 0
\end{array}\right]\right\| \leq\left\|\left[\begin{array}{cccc}
S_{1} & S_{2} & \cdots & S_{n} \\
0 & 0 & \cdots & 0 \\
\vdots & \vdots & & \vdots \\
0 & 0 & \cdots & 0
\end{array}\right]\right\| .
$$

Hence, $\left\|\sum_{i=1}^{n} A_{i} A_{i}^{*}\right\| \leq\left\|\sum_{i=1}^{n} S_{i} S_{i}^{*}\right\|=1$. This completes the proof. 
Let us remark that if $A_{1}, A_{2}, \ldots, A_{n} \in B(\mathcal{H})$ such that the map

$$
\Phi: \mathcal{P}_{n} \rightarrow B(\mathcal{H}) ; \quad \Phi(p)=p\left(A_{1}, \ldots, A_{n}\right)
$$

is completely contractive, one can get another proof for the existence of an isometric dilation for $A_{1}, A_{2}, \ldots, A_{n}$ (see [Po2]).

Indeed, according to [Arv, Prop. 1.2.8] $\Phi$ has a unique completely positive extention $\tilde{\Phi}$ to the closure of $\mathcal{P}_{n}+\mathcal{P}_{n}^{*}$ such that

$$
\tilde{\Phi}\left(p+q^{*}\right)=p\left(A_{1}, \ldots, A_{n}\right)+q\left(A_{1}, \ldots, A_{n}\right)^{*}
$$

for any $p, q \in \mathcal{P}_{n} \subset C^{*}\left(S_{1}, \ldots, S_{n}\right)$. Here $\mathcal{P}_{n}^{*}$ stands for $\left\{p\left(S_{1}, \ldots, S_{n}\right)^{*} ; p \in \mathcal{P}_{n}\right\}$. Using the extension theorem of Arveson $[\mathrm{Arv}]$ we infer that there is a completely positive linear map $\Psi: C^{*}\left(S_{1}, \ldots, S_{n}\right) \rightarrow B(\mathcal{H})$ such that

$$
\left.\Psi\right|_{\overline{\mathcal{P}_{n}+\mathcal{P}_{n}^{*}}}=\tilde{\Phi} .
$$

According to Stinespring's theorem [S]

$$
\Psi(f)=V^{*} \pi(f) V, \quad \text { for any } f \in C^{*}\left(S_{1}, \ldots, S_{n}\right),
$$

where $\pi$ is a *-representation of $C^{*}\left(S_{1}, \ldots, S_{n}\right)$ on some Hilbert space $\mathcal{K}$ and $V$ is a bounded operator from $\mathcal{H}$ to $\mathcal{K}$. Since $\Psi(1)=I$, it follows that $V^{*} V=I$, that is, $V$ is an isometric embedding of $\mathcal{H}$ in $\mathcal{K}$. Using $V$, we can identify $\mathcal{H}$ with a subspace of $\mathcal{K}$, and $(2.5)$ becomes $\Psi(f)=\left.P_{\mathcal{H}} \pi(f)\right|_{\mathcal{H}}, P_{\mathcal{H}}$ being the projection of $\mathcal{K}$ on $\mathcal{H}$. Since

$$
\pi\left(S_{i}\right)^{*} \pi\left(S_{j}\right)=\pi\left(S_{i}^{*} S_{j}\right)= \begin{cases}0, & \text { if } i \neq j, \\ I_{\mathcal{K}}, & \text { if } i=j,\end{cases}
$$

it follows that $\left\{\pi\left(S_{i}\right)\right\}_{i=1}^{n}$ are isometries with orthogonal ranges. On the other hand, the relations $(2.3),(2.4),(2.5)$ imply

$$
p\left(A_{1}, \ldots, A_{n}\right)=\left.P_{\mathcal{H}} p\left(\pi\left(S_{1}\right), \ldots, \pi\left(S_{n}\right)\right)\right|_{\mathcal{H}}, \quad \text { for any } i=1,2, \ldots, n \text { and } p \in \mathcal{P}
$$

that is, $\left\{\pi\left(S_{i}\right)\right\}_{i=1}^{n}$ is an isometric dilation of $\left\{A_{i}\right\}_{i=1}^{n}$.

Corollary 2.2. $\left[A_{1}, \ldots, A_{n}\right]$ is a contraction if and only if the map

$$
\Phi: C^{*}\left(S_{1}, \ldots, S_{n}\right) \rightarrow B(\mathcal{H}) ; \quad \Phi\left(S_{i_{1}} \cdots S_{i_{k}} S_{j_{1}}^{*} \cdots S_{j_{p}}^{*}\right)=A_{i_{1}} \cdots A_{i_{k}} A_{j_{1}}^{*} \cdots A_{j_{p}}^{*},
$$

$1 \leq i_{1}, \ldots, i_{k}, j_{1}, \ldots, j_{p} \leq n$, is a completely contractive linear map.

Corollary 2.3. $\left[A_{1}, \ldots, A_{n}\right]$ is a contraction if and only if there is a completely positive linear map $\Phi: C^{*}\left(S_{1}, \ldots, S_{n}\right) \rightarrow B(\mathcal{H})$ such that $\Phi\left(S_{i}\right)=A_{i}$, for $i=$ $1,2, \ldots, n$.

In [P1] V. Paulsen proved that an operator $A \in B(\mathcal{H})$ is similar to a contraction if and only if it is completely polynomially bounded. In what follows we will extend this result to our setting. 
Theorem 2.4. Let $A_{1}, \ldots, A_{n}$ be in $B(\mathcal{H})$. The following statements are equivalent:

(i) There exist a contraction $\left[T_{1}, \ldots, T_{n}\right]$ and an invertible operator $S$ such that

$$
A_{i}=S^{-1} T_{i} S, \quad \text { for any } i=1,2, \ldots, n .
$$

(ii) The sequence $A_{1}, \ldots, A_{n}$ is c.p.b.

Proof. Assume (i) holds. We have

$$
\Phi(p)=p\left(A_{1}, \ldots, A_{n}\right)=S p\left(T_{1}, \ldots, T_{n}\right) S^{-1} \text { for any } p \in \mathcal{P}_{n} .
$$

Since the map

$$
\Psi: \mathcal{P}_{n} \rightarrow B(\mathcal{H}) ; \quad \Psi(p)=p\left(T_{1}, \ldots, T_{n}\right)
$$

is completely contractive by Theorem 2.1 , it follows that

$$
\|\Phi\|_{c b} \leq\|S\|\|\Psi\|_{c b}\left\|S^{-1}\right\|<\infty .
$$

Conversely, assume that (ii) holds. Since $\Phi: \mathcal{A}_{n} \rightarrow B(\mathcal{H}) ; \quad \Phi(f)=f\left(A_{1}, \ldots, A_{n}\right)$ is completely bounded, according to [P1] there is an invertible operator $S$ such that

$$
\mathcal{A}_{n} \in f \rightarrow S \Phi(f) S^{-1}=f\left(S A_{1} S^{-1}, \ldots, S A_{n} S^{-1}\right) \in B(\mathcal{H})
$$

is completely contractive. Now, Theorem 2.1 shows that $\left[S A_{1} S^{-1}, \ldots, S A_{n} S^{-1}\right]$ is a contraction. Setting $S A_{i} S^{-1}=T_{i}, i=1,2, \ldots, n$, the proof is complete.

Corollary 2.5. A representation $\Phi: \mathcal{A}_{n} \rightarrow B(\mathcal{H})$ is c.b. if and only if it is given by $\Phi\left(S_{i}\right)=S T_{i} S^{-1}, i=1, \ldots, n$, where $\left[T_{1}, \ldots, T_{n}\right]$ is a contraction and $S$ is an invertible operator.

Let $A_{1}, \ldots, A_{n}$ be a sequence of operators on $\mathcal{H}$. Following [B2] we define the "spectral radius" of this sequence as being

$$
r\left(A_{1}, \ldots, A_{n}\right):=\lim _{k \rightarrow \infty}\left\|\sum_{|f|=k} A_{f} A_{f}^{*}\right\|^{1 / 2 k},
$$

where for any $f=\left(i_{1}, \ldots, i_{k}\right), 1 \leq i_{1}, \ldots, i_{k} \leq n, A_{f}$ stands for the product $A_{i_{1}} \cdots A_{i_{k}}$ and $|f|=k$.

Proposition 2.6. Let $A_{1}, \ldots, A_{n}$ be in $B(\mathcal{H})$. If the map

$$
\Phi: \mathcal{P}_{n} \rightarrow B(\mathcal{H}) ; \Phi=p\left(A_{1}, \ldots, A_{n}\right)
$$

is c.b., then $r\left(A_{1}, \ldots, A_{n}\right) \leq 1$.

Proof. According to [Po1, Prop.3.5]

$$
r\left(A_{1}, \ldots, A_{n}\right)=\inf _{S}\left\|\sum_{i=1}^{n}\left(S A_{i} S^{-1}\right)\left(S A_{i} S^{-1}\right)^{*}\right\|^{1 / 2}
$$

where the infimum is taken over all invertible operators on $\mathcal{H}$. By Theorem 2.4 there exist a contraction $\left[T_{1}, \ldots, T_{n}\right]$ and an invertible operator $S$ such that $A_{i}=S^{-1} T_{i} S$ for any $i=1,2, \ldots, n$. Therefore, $r\left(A_{1}, \ldots, A_{n}\right) \leq\left\|\sum T_{i} T_{i}^{*}\right\|^{1 / 2} \leq 1$. 
Propositon 2.7. Let $A_{1}, \ldots, A_{n} \in B(\mathcal{H})$ such that one of the following statements holds:

(i) There exists $b>a>0$ such that

$$
a\|h\|^{2} \leq \sum_{|f|=k}\left\|A_{f}^{*} h\right\|^{2} \leq b\|h\|^{2} \quad \text { for any } h \in \mathcal{H} \text { and } k=1,2, \ldots
$$

(ii) $r\left(A_{1}, \ldots, A_{n}\right)<1$.

Then, the map $\Phi: \mathcal{P}_{n} \rightarrow B(\mathcal{H}) ; \Phi(p)=p\left(A_{1}, \ldots, A_{n}\right)$ is c.b.

Proof. If either one of the above statements holds, then, according to [Po1], there exist a contraction $\left[T_{1}, \ldots, T_{n}\right]$ and an invertible operator $S$ such that $A_{i}=S^{-1} T_{i} S$, $i=1,2, \ldots, n$. According to Theorem 2.4 the result follows.

Let us remark that if (i) holds, then $r\left(A_{1}, \ldots, A_{n}\right)=1$. All the results of this section can be easily extended to $n=\infty$.

\section{The non-COMmutative Disc algebras $\mathcal{A}_{n}$}

Let $V_{1}, \ldots, V_{n}$ be isometries on a Hilbert space $\mathcal{K}$ such that $V_{1} V_{1}^{*}+\cdots+V_{n} V_{n}^{*} \leq$ $I_{\mathcal{K}}$. Let $\operatorname{Alg}\left(V_{1}, \ldots, V_{n}\right)$ denote the smallest closed subalgebra of $B(\mathcal{K})$ containing $I_{\mathcal{K}}, V_{1}, \ldots, V_{n}$. This algebra is the closure in the uniform norm of the collection of polynomials in $V_{1}, \ldots, V_{n}$, that is,

$$
\operatorname{Alg}\left(V_{1}, \ldots, V_{n}\right)=\operatorname{clos}\left\{p\left(V_{1}, \ldots, V_{n}\right): p \in \mathcal{P}_{n}\right\} .
$$

In [Po3] we proved that if $S_{1}, \ldots, S_{n}$ are the left creation operators on the Fock space $F^{2}\left(H_{n}\right)$, then the Banach algebras $\operatorname{Alg}\left(S_{1}, \ldots, S_{n}\right)$ and $\mathcal{A}_{n}$ are isometrically isomorphic. In what follows we will obtain a more general result.

Theorem 3.1. The Banach algebras $\operatorname{Alg}\left(V_{1}, \ldots, V_{n}\right)$ and $\mathcal{A}_{n}(n=2,3, \ldots, \infty)$ are completely isometrically isomorphic.

Proof. Consider the case $n=2,3, \ldots$ Suppose first that $V_{1}, \ldots, V_{n}$ are isometries on $\mathcal{K}$ such that $V_{1} V_{1}^{*}+\cdots+V_{n} V_{n}^{*}=I_{\mathcal{K}}$. According to the von Neumann inequality (1.2) we have

$$
\left\|p\left(V_{1}, \ldots, V_{n}\right)\right\| \leq\left\|p\left(S_{1}, \ldots, S_{n}\right)\right\|, \text { for any } p \in \mathcal{P}_{n} .
$$

On the other hand, according to [Po2, Prop.2.6] there is a sequence $W_{1}, \ldots, W_{n}$ of isometries on a Hilbert space $\mathcal{G} \supset \mathcal{K}$ such that

$$
\sum_{i=1}^{n} W_{i} W_{i}^{*}=I_{\mathcal{G}} \text { and } S_{i_{1}} \cdots S_{i_{k}}=\left.P_{\mathcal{K}} W_{i_{1}} \cdots W_{i_{k}}\right|_{\mathcal{K}}
$$

for any $1 \leq i_{1}, \ldots, i_{k} \leq n$. Hence we deduce that

$$
p\left(S_{1}, \ldots, S_{n}\right)=\left.P_{\mathcal{K}} p\left(W_{1}, \ldots, W_{n}\right)\right|_{\mathcal{K}}, \quad p \in \mathcal{P}_{n},
$$

and

$$
\left\|p\left(S_{1}, \ldots, S_{n}\right)\right\| \leq\left\|p\left(W_{1}, \ldots, W_{n}\right)\right\| .
$$


Since the Cuntz algebra $\mathcal{O}_{n}$ does not depend on the generators $[\mathrm{Cu}]$, we have that

$$
\left\|p\left(V_{1}, \ldots, V_{n}\right)\right\|=\left\|p\left(W_{1}, \ldots, W_{n}\right)\right\|,
$$

which together with (3.1) and (3.2) shows that

$$
\left\|p\left(V_{1}, \ldots, V_{n}\right)\right\|=\left\|p\left(S_{1}, \ldots, S_{n}\right)\right\|, \text { for any } p \in \mathcal{P}_{n} .
$$

In the case when $V_{1} V_{1}^{*}+\cdots+V_{n} V_{n}^{*} \leq I_{\mathcal{K}}$, the relation (3.4) holds also according to [Po5]. This relation shows that the map $V_{i} \rightarrow S_{i}(i=1,2, \ldots, n)$ extends to an isometric isomorphism from $\operatorname{Alg}\left(V_{1}, \ldots, V_{n}\right)$ onto $\mathcal{A}_{n}$. The completely isometric (in short c.i.) part follows in the same way, passing to matrices. The case $n=\infty$ follows from $[\mathrm{Cu}]$. This completes the proof.

Let $V_{1}, V_{2}, \ldots$ be a sequence of isometries satisfying $\sum_{i=1}^{k} V_{i} V_{i}^{*} \leq I$ for every $k \in \mathbb{N}$. According to Theorem 3.1 we have $A l g\left(V_{1}, \ldots, V_{k}\right) \stackrel{\text { c.i. }}{\simeq} \mathcal{A}_{k}$ for any $k=1,2, \ldots$ and $\operatorname{Alg}\left(V_{1}, V_{2}, \ldots\right) \stackrel{\text { c.i. }}{\simeq} \mathcal{A}_{\infty}$. Thus, it is clear that

$$
\mathcal{A}_{2} \stackrel{\text { c.i. }}{\subset} \mathcal{A}_{3} \stackrel{\text { c.i. }}{\subset} \cdots \stackrel{\text { c.i. }}{\subset} A_{\infty} .
$$

On the other hand, consider $\mathcal{A}_{2}=\operatorname{Alg}\left(S_{1}, S_{2}\right)$. If we put

$$
V_{1}=S_{1}, V_{2}=S_{2} S_{1}, \ldots, V_{k}=S_{2}^{k-1} S_{1}, \ldots,
$$

then $\sum_{i=1}^{k} V_{i} V_{i}^{*} \leq I$ for every $k \in \mathbb{N}$. By Theorem 3.1 we have

$$
\mathcal{A}_{\infty} \stackrel{\text { c.i. }}{\simeq} \operatorname{Alg}\left(V_{1}, V_{2}, \ldots\right) \subset \operatorname{Alg}\left(S_{1}, S_{2}\right) \stackrel{\text { c.i. }}{\simeq} \mathcal{A}_{2} \text { and } \mathcal{A}_{3} \stackrel{\text { c.i. }}{\simeq} \operatorname{Alg}\left(V_{1}, V_{2}, V_{3}\right) \subset \mathcal{A}_{2} .
$$

By induction we get the following chain of inclusions:

$$
\mathcal{A}_{2} \stackrel{\text { c.i. }}{\supset} A_{3} \stackrel{\text { c.i. }}{\supset} \stackrel{\text { c.i. }}{\supset} \mathcal{A}_{\infty}
$$

In [A] A. Arias showed that, as Banach spaces, the disc algebras are completely isomorphic. In what follows we will show that they are not isomorphic as Banach algebras.

Let $\lambda=\left(\lambda_{1}, \ldots, \lambda_{n}\right)$ be in $\overline{\left(\mathbb{C}^{n}\right)_{1}}=\left\{\left(\lambda_{1}, \ldots \lambda_{n}\right):\left|\lambda_{1}\right|^{2}+\cdots+\left|\lambda_{n}\right|^{2} \leq 1\right\}$, and define the "evaluation" functional

$$
\Phi_{\lambda}: \mathcal{P}_{n} \rightarrow \mathbb{C} ; \quad \Phi_{\lambda}(p)=p\left(\lambda_{1}, \ldots, \lambda_{n}\right) .
$$

According to the von Neumann inequality (1.2) we have

$$
\left|p\left(\lambda_{1}, \ldots, \lambda_{n}\right)\right|=\left\|p\left(\lambda_{1} I_{\mathbb{C}}, \ldots, \lambda_{n} I_{\mathbb{C}}\right)\right\| \leq\left\|p\left(S_{1}, \ldots, S_{n}\right)\right\|=\|p\|_{\infty} .
$$

Hence, $\Phi_{\lambda}$ has a unique extension to the disc algebra $\mathcal{A}_{n}$. Therefore $\Phi$ is a character of $\mathcal{A}_{n}$. Let $M_{\mathcal{A}_{n}}$ be the set of all characters of $\mathcal{A}_{n}$ and let $\Psi: \overline{\left(\mathbb{C}^{n}\right)_{1}} \rightarrow M_{\mathcal{A}_{n}}$ be defined by $\Psi(\lambda)=\Phi_{\lambda}$. 
Theorem 3.2. $\Psi$ is a homeomorphism of $\overline{\left(\mathbb{C}^{n}\right)_{1}}$ onto $M_{\mathcal{A}_{n}} \quad(n=2,3, \ldots)$.

Proof. Let us show that $\Psi$ is one-to-one. If $\lambda=\left(\lambda_{1}, \ldots, \lambda_{n}\right)$ and $\mu=\left(\mu_{1}, \ldots, \mu_{n}\right)$ are in $\overline{\left(\mathbb{C}^{n}\right)}$, then $\Psi(\lambda)=\Psi(\mu)$ implies that

$$
\lambda_{i}=\Phi_{\lambda}\left(S_{i}\right)=\Phi_{\mu}\left(S_{i}\right)=\mu_{i} \text {, for any } i=1,2, \ldots, n .
$$

Therefore $\lambda=\mu$. Now, assume that $\Phi: \mathcal{A}_{n} \rightarrow \mathbb{C}$ is a character. Setting $\Phi\left(S_{i}\right)=$ $\lambda_{i}, i=1,2, \ldots, n$, we have

$$
\Phi\left(p\left(S_{1}, \ldots, S_{n}\right)\right)=p\left(\lambda_{1}, \ldots, \lambda_{n}\right), \text { for any } p \in \mathcal{P}_{n} .
$$

Since $\Phi$ is a character, it follows that it is completely contractive. Applying Theorem 2.1 when $A_{i}=\lambda_{i} I_{\mathbb{C}}, i=1,2, \ldots, n$, we infer that $\left[\lambda_{1} I_{\mathbb{C}}, \ldots, \lambda_{n} I_{\mathbb{C}}\right]$ is a contraction, i.e., $\left|\lambda_{1}\right|^{2}+\cdots+\left|\lambda_{n}\right|^{2} \leq 1$. Moreover the identity

$$
\Phi\left(p\left(S_{1}, \ldots, S_{n}\right)\right)=p\left(\lambda_{1}, \ldots, \lambda_{n}\right)=\Phi_{\lambda}\left(p\left(S_{1}, \ldots, S_{n}\right)\right)
$$

proves that $\Phi$ agrees with $\Phi_{\lambda}$ on the dense subset $\mathcal{P}_{n}$ of $\mathcal{A}_{n}$, therefore $\Phi=\Phi_{\lambda}$.

Since both $\overline{\left(\mathbb{C}^{n}\right)_{1}}$ and $M_{\mathcal{A}_{n}}$ are compact Hausdorff spaces and $\Psi$ is one-to-one and onto, to complete the proof it suffices to show that $\Psi$ is continuous.

Suppose that $\lambda^{\alpha}=\left(\lambda_{1}^{\alpha}, \ldots, \lambda_{n}^{\alpha}\right), \alpha \in J$, is a net in $\overline{\left(\mathbb{C}^{n}\right)}$, such that $\lim _{\alpha \in J} \lambda^{\alpha}=$ $\lambda=\left(\lambda_{1}, \ldots, \lambda_{n}\right)$. Since $\sup _{\alpha \in J}\left\|\Phi_{\lambda^{\alpha}}\right\| \leq 1$ and $\mathcal{P}_{n}$ is dense in $\mathcal{A}_{n}$, and since

$$
\lim _{\alpha \in J} \Phi_{\lambda^{\alpha}}\left(p\left(S_{1}, \ldots, S_{n}\right)\right)=\lim _{\alpha \in J} p\left(\lambda_{1}^{\alpha}, \ldots, \lambda_{n}^{\alpha}\right)=p\left(\lambda_{1}, \ldots, \lambda_{n}\right)=\Phi_{\lambda}\left(p\left(S_{1}, \ldots, S_{n}\right)\right)
$$

for every $p \in \mathcal{P}_{n}$, it follows that $\Psi$ is continuous. The proof is complete.

Remark 3.3. The above theorem can be easily extended to the case $n=\infty$ in a slightly adapted version, showing that $\overline{\left(\ell^{2}\right)}$ ( with the weak topology) is homeomorphic to $M_{\mathcal{A}_{\infty}}$.

The Cuntz algebra $\mathcal{O}_{n}$ is uniquely defined as the $C^{*}$-algebra generated by $n=$ $2,3, \ldots$ isometries satisfying

$$
s_{i}^{*} s_{j}=\delta_{i j} 1, \quad \sum_{j=1}^{n} s_{i} s_{j}^{*}=1
$$

$[\mathrm{Cu}]$. We define $\mathcal{O}_{1}=C(\mathbb{T})$ (see $[\mathrm{C}]$ ), and $\mathcal{O}_{\infty}$ as the $C^{*}$-algebra generated by isometries $s_{1}, s_{2}, \ldots$ satisfying merely the first relation in (3.8). Notice that the disc algebra $\mathcal{A}_{n}$ can be viewed as a subalgebra of the Cuntz algebra $\mathcal{O}_{n}$. Indeed, if $s_{1}, \ldots, s_{n}$ is a system of generators for $\mathcal{O}_{n}$, then according to Theorem $3.1 \mathcal{A}_{n} \stackrel{\text { c.i. }}{\simeq}$ $\operatorname{Alg}\left(s_{1}, \ldots, s_{n}\right) \subset \mathcal{O}_{n}(n=2,3, \ldots)$ and $\mathcal{A}_{\infty} \stackrel{\text { c.i. }}{\simeq} \operatorname{Alg}\left(s_{1}, s_{2}, \ldots\right) \subset \mathcal{O}_{\infty}$.

It was proved by Pimsner and Popa [PP] that if $n \neq m$, then $\mathcal{O}_{n} \neq \mathcal{O}_{m}$ (see also [PS]). Using Theorem 3.2 and the dimension theory $[\mathrm{HW}]$ it is easy to get the following.

Corollary 3.4. The Banach algebras $\mathcal{A}_{n}$ and $\mathcal{A}_{m}$ are not isomorphic if $n \neq m$, $n, m=1,2, \ldots, \infty$.

On the other hand, since $\mathcal{O}_{n}$ has no non-trivial character, it follows that $\mathcal{A}_{n} \not$ $\mathcal{O}_{m}$ for any $n, m=2, \ldots, \infty$. 


\section{Disc Algebras And COHOMOlogy}

Let $A$ be a complex Banach algebra with unit, $X$ a Banach $A$-bimodule, and $X^{\prime}$ the dual Banach $A$-bimodule (see $[\mathrm{BD}]$ ). We need to recall from $[\mathrm{BD}]$ a few definitions.

A bounded $X$-derivation is a bounded linear mapping $D$ of $A$ into $X$ such that

$$
D(a b)=(D a) b+a(D b), \quad \text { for any } a, b \in A .
$$

The set of all bounded $X$-derivations is denoted by $Z^{1}(A, X)$. For each $x \in X$ let us define $\delta_{x}: A \rightarrow X$ by $\delta_{x}(a)=a x-x a$. We call $\delta_{x}$ an inner $X$-derivation, and denote by $B^{1}(A, X)$ the set of all inner $X$-derivations. The quotient space $Z^{1}(A, X) / B^{1}(A, X)$ is called the first cohomology group of $A$ with coefficients in $X$, and it is denoted by $H^{1}(A, X)$. A Banach algebra $A$ is said to be amenable if $H^{1}\left(A, X^{\prime}\right)=\{0\}$ for every Banach $A$-bimodule $X$.

In what follows we shall show that the disc algebras $\mathcal{A}_{n}(n=2,3, \ldots, \infty)$ are not amenable.

Of course $\mathbb{C}$, the set of all complex numbers, is a Banach $\mathcal{A}_{n}$-bimodule under the module multiplication

$$
\lambda \cdot f=f \cdot \lambda=\lambda f(0)
$$

where for each $f \in \mathcal{A}_{n}, f(0):=\Phi_{(0, \ldots, 0)}(f)$ (see the relation (3.7)). Notice that $|\lambda \cdot f| \leq|\lambda|\|f\|_{\infty}, \quad$ for any $\lambda \in \mathbb{C}, f \in \mathcal{A}_{n}$.

Theorem 4.1. The first cohomology group of $\mathcal{A}_{n}(n=2,3, \ldots)$ with coefficients in $\mathbb{C}$ is isomorphic to the additive group $\mathbb{C}^{n}$, i.e., $H^{1}\left(\mathcal{A}_{n}, \mathbb{C}\right) \simeq\left(\mathbb{C}^{n},+\right)$. If $n=\infty$, then $H^{1}\left(\mathcal{A}_{\infty}, \mathbb{C}\right) \simeq\left(\ell^{2},+\right)$.

Proof. It is clear that $B^{1}\left(\mathcal{A}_{n}, \mathbb{C}\right)=\{0\}$. If $D \in Z^{1}\left(\mathcal{A}_{n}, \mathbb{C}\right)$, then, using (4.1) and (4.2) it is easy to see that $D(1)=0$ and $D\left(e_{i_{1}} \otimes \cdots \otimes e_{i_{k}}\right)=0$ for $k=$ $2,3, \ldots$ Therefore the derivation $D$ is determined by $D\left(e_{1}\right), D\left(e_{2}\right), \ldots, D\left(e_{n}\right)$. Let $D\left(e_{i}\right)=\lambda_{i} \in \mathbb{C}, i=1,2, \ldots, n$. Since $D$ is a linear mapping for each $f \in \mathcal{A}_{n}, f=$ $a_{0}+\sum_{i=1}^{n} a_{i} e_{i}+\cdots$, we have $D(f)=\sum_{i=1}^{n} a_{i} \lambda_{i}$. It is easy to see that

$$
D(f \otimes g)=D(f) g+f D(g), \quad \text { for any } f, g \in \mathcal{A}_{n} .
$$

Let us show that $D$ is bounded if and only if $\sum_{i=1}^{n}\left|\lambda_{i}\right|^{2}<\infty(n=2,3, \ldots, \infty)$. For each $f \in \mathcal{A}_{n}, f=a_{0}+\sum_{i=1}^{n} a_{i} e_{i}+\cdots$, we have

$$
\begin{aligned}
|D(f)| & =\left|\sum_{i=1}^{n} a_{i} \lambda_{i}\right| \leq\left(\sum_{i=1}^{n}\left|a_{i}\right|^{2}\right)^{1 / 2}\left(\sum_{i=1}^{n}\left|\lambda_{i}\right|^{2}\right)^{1 / 2} \\
& \leq\|f\|_{2}\left(\sum_{i=1}^{n}\left|\lambda_{i}\right|^{2}\right)^{1 / 2} \leq\|f\|_{\infty}\left(\sum_{i=1}^{n}\left|\lambda_{i}\right|^{2}\right)^{1 / 2} .
\end{aligned}
$$


Therefore $D$ is a bounded $\mathbb{C}$-derivation. We need to prove the converse only for $n=\infty$. Suppose that $D: \mathcal{A}_{\infty} \rightarrow \mathbb{C}$ is a bounded derivation. For each $f \in \mathcal{A}_{\infty}, f=$ $a_{0}+\sum_{i=1}^{n} a_{i} e_{i}+\cdots, D(f)=\sum_{i=1}^{\infty} a_{i} \lambda_{i}$ for some $\lambda_{i} \in \mathbb{C}, i=1,2, \ldots$, and

$$
|D(f)| \leq M\|f\|_{\infty}, \quad \text { for any } f \in \mathcal{A}_{\infty} .
$$

In particular, for any $\left\{a_{i}\right\}_{i=1}^{\infty} \in \ell^{2}, g=\sum_{i=1}^{\infty} a_{i} e_{i}$ is in $\mathcal{A}_{\infty}$ and $\|g\|_{\infty}=\|g\|_{2}$. In this case the relation (4.3) shows that

$$
\left|\sum_{i=1}^{\infty} a_{i} \lambda_{i}\right| \leq M\left\|\left\{a_{i}\right\}_{i=1}^{\infty}\right\|_{2},
$$

for any $\left\{a_{i}\right\}_{i=1}^{\infty} \in \ell^{2}$. Hence we deduce that $\left\{\lambda_{i}\right\}_{i=1}^{\infty} \in \ell^{2}$.

Now it is clear that $H^{1}\left(\mathcal{A}_{n}, \mathbb{C}\right) \simeq\left(\mathbb{C}^{n},+\right)$ for $n=2,3, \ldots$, and $H^{1}\left(\mathcal{A}_{\infty}, \mathbb{C}\right) \simeq$ $\left(\ell^{2},+\right)$.

Since $\mathbb{C}$ is a dual bimodule, we have the following.

Corollary 4.2. The disc algebras $\mathcal{A}_{n}(n=2,3, \ldots, \infty)$ are not amenable.

A similar proof to that of Theorem 4.1 shows the following.

Remark 4.3. $H^{1}\left(F_{n}^{\infty}, \mathbb{C}\right) \simeq\left(\mathbb{C}^{n},+\right)$ for $n=2,3, \ldots$, and $H^{1}\left(F_{\infty}^{\infty}, \mathbb{C}\right) \simeq\left(\ell^{2},+\right)$.

Corollary 4.4. The non-commutative Hardy algebras $F_{n}^{\infty}(n=2,3, \ldots, \infty)$ are not amenable. Moreover, if $n \neq m, n, m=1,2, \ldots, \infty$, then $F_{n}^{\infty}$ and $F_{m}^{\infty}$ are not Banach isomorphic.

\section{The Right Joint Spectrum of $\left(S_{1}, \ldots, S_{n}\right)$}

If $A=\left(A_{1}, \ldots, A_{n}\right)$ is an $n$-tuple of operators acting on $\mathcal{H}$, then the joint left (resp. right) spectrum of $A$ is the set of $n$-tuples of complex numbers $\lambda=$ $\left(\lambda_{1}, \lambda_{2}, \ldots, \lambda_{n}\right)$ such that the left (resp. right) ideal of $B(\mathcal{H})$ generated by the set $\left\{\lambda_{1} I-A_{1}, \lambda_{2} I-A_{2}, \ldots, \lambda_{n} I-A_{n}\right\}$ does not contain the identity operator (see [B1], [B2]). Let us denote the left (resp. right) spectrum of $A$ by $\sigma_{l}(A)$ (resp. $\sigma_{r}(A)$ ). Let $S_{1}, \ldots, S_{n}$ be the left creation operators on the full Fock space $F^{2}\left(H_{n}\right)$.

Theorem 5.1. $\sigma_{r}\left(S_{1}, \ldots, S_{n}\right)={\overline{\left(\mathbb{C}^{n}\right)}}_{1}$.

Proof. Let $\mu=\left(\mu_{1}, \ldots, \mu_{n}\right) \in \overline{\left(\mathbb{C}^{n}\right)}$. Suppose that there is $\delta>0$ such that

$$
\sum_{i=1}^{n}\left\|\left(S_{i}-\mu_{i} I\right)^{*} h\right\|^{2} \geq \delta\|h\|^{2}, \quad \text { for any } h \in F^{2}\left(H_{n}\right) .
$$

For

$$
h=1+\sum_{k=1}^{\infty} \sum_{1 \leq i_{1}, \ldots, i_{k} \leq n} \lambda_{i_{1}} \cdots \lambda_{i_{k}} e_{i_{1}} \otimes \cdots \otimes e_{i_{k}},
$$

where $\left(\lambda_{1}, \ldots, \lambda_{2}\right) \in\left(\mathbb{C}^{n}\right)_{1}$, we have $S_{i}^{*} h=\lambda_{i} h, \quad i=1,2, \ldots, n$, and

$$
\sum_{i=1}^{n}\left\|\left(S_{i}-\mu_{i} I\right)^{*} h\right\|^{2}=\sum_{i=1}^{n}\left|\lambda_{i}-\bar{\mu}_{i}\right|^{2}\|h\|^{2} .
$$


The relation (5.1) becomes

$$
\sum_{i=1}^{n}\left|\lambda_{i}-\bar{\mu}_{i}\right|^{2} \geq \delta, \text { for any }\left(\lambda_{1}, \ldots, \lambda_{n}\right) \in\left(\mathbb{C}^{n}\right)_{1} .
$$

Since (5.2) is not true, according to the Corona-type theorem [Po4] it does not exist $\left\{B_{1}, \ldots, B_{n}\right\} \subset B\left(F^{2}\left(H_{n}\right)\right)$ such that

$$
\left(S_{1}-\mu_{1} I\right) B_{1}+\cdots+\left(S_{n}-\mu_{n} I\right) B_{n}=I .
$$

Therefore $\overline{\left(\mathbb{C}^{n}\right)_{1}} \subset \sigma_{r}\left(S_{1}, \ldots, S_{n}\right)$.

Conversely, let $\left(\lambda_{1}, \ldots, \lambda_{n}\right) \in \sigma_{r}\left(S_{1}, \ldots, S_{n}\right)$. This means that the left ideal of $B\left(F^{2}\left(H_{n}\right)\right)$ generated by $\left\{S_{1}^{*}-\bar{\lambda}_{1} I, \ldots, S_{n}^{*}-\bar{\lambda}_{n} I\right\}$ does not contain the identity. According to [D, Theorem 2.9.5] there is a state $\sigma$ on $B\left(F^{2}\left(H_{n}\right)\right)$ such that $\sigma\left(X S_{i}^{*}\right)=\bar{\lambda}_{i} \sigma(X)$ for each $X \in B\left(F^{2}\left(H_{n}\right)\right)$ and $i=1,2, \ldots, n$. Then, for any $k=1,2, \ldots$,

$$
\left|\sigma\left(\sum_{|f|=k} S_{f} S_{f}^{*}\right)\right| \leq\left\|\sum_{|f|=k} S_{f} S_{f}^{*}\right\|=1 .
$$

Hence, $\sum_{|f|=k}\left\|\lambda_{f}\right\|^{2} \leq 1$. Since $\left(\sum_{j=1}^{n}\left|\lambda_{j}\right|^{2}\right)^{k}=\sum_{|f|=k}\left|\lambda_{f}\right|^{2}$, it follows that $\left(\lambda_{1}, \ldots, \lambda_{n}\right)$ $\in{\overline{\left(\mathbb{C}^{n}\right)}}_{1}$. This completes the proof.

Using Theorem 3.2 we infer the following.

Remark 5.2. (1) $\sigma_{r}\left(S_{1}, \ldots, S_{n}\right)=\left\{\left(\Phi\left(S_{1}\right), \ldots, \Phi\left(S_{n}\right)\right): \Phi \in M_{\mathcal{A}_{n}}\right\}$.

(2) If $f_{1}, \ldots, f_{n} \in \mathcal{A}_{n}$, then $\sigma_{r}\left(f_{1}, \ldots, f_{n}\right) \supset\left\{\left(\Phi\left(f_{1}\right), \ldots, \Phi\left(f_{n}\right)\right): \Phi \in M_{\mathcal{A}_{n}}\right\}$.

\section{Open PRoBlems}

Let $\lambda=\left(\lambda_{1}, \ldots, \lambda_{n}\right) \in\left(\mathbb{C}^{n}\right)_{1}$ and let $\Phi_{\lambda}: F_{n}^{\infty} \rightarrow \mathbb{C}$ be defined by $\Phi(f)=$ $\left\langle f, z_{\lambda}\right\rangle, f \in F^{\infty}$, where $z_{\lambda}=1+\sum_{k=1}^{\infty}\left(\lambda_{1} e_{1}+\cdots+\lambda_{n} e_{n}\right)^{k}$. One can prove that $\Phi_{\lambda}$ is a multiplicative functional on $F_{n}^{\infty}$.

Problem 6.1. Is $\left\{\Phi_{\lambda}: \lambda \in\left(\mathbb{C}^{n}\right)_{1}\right\} w^{*}$-dense in the set of all multiplicative functionals on $F_{n}^{\infty}$ ?

In [Arv] W. Arveson proved that if $T$ is a contraction on a Hilbert space, then $T$ gives rise to a completely isometric representation of the disc algebra if and only if the spectrum of $T$ contains the unit disc.

Problem 6.2. Characterize the contractions $\left[T_{1}, \ldots, T_{n}\right]$ for which the map

$$
\mathcal{A}_{n} \in p \mapsto p\left(T_{1}, \ldots, T_{n}\right) \in B(\mathcal{H})
$$

is a completely isometric representation of the disc algebra $\mathcal{A}_{n}$.

According to [Po3], [Po5] and Theorem 3.1, one can show that if $T_{i}=A_{i} \oplus$ $V_{i}$, where $V_{i}, i=1,2, \ldots, n$, are isometries, then the above map is a completely isometric representation.

Let $\Phi: \mathcal{A}_{n} \rightarrow B(\mathcal{H})$ defined by $\Phi(p)=p\left(A_{1}, \ldots, A_{n}\right)$, where $A_{1}, \ldots, A_{n} \in B(\mathcal{H})$.

Problem 6.3. Is the implication, $\Phi$ contractive $\Longrightarrow \Phi$ completely contractive, true? 


\section{ACKNOWLEDGMENTS}

The author thanks Gilles Pisier for useful discussions on the subject of this paper.

\section{REFERENCES}

[A] A. Arias, Completely bounded isomorphisms of operator algebras, Proc.Amer.Math.Soc. 124 (1966), 1091-1101.

[Arv] W.B. Arveson, Subalgebras of $C^{*}$-algebras, Acta.Math. 123 (1969), 141-224. MR 40:6274

[BD] F.F. Bonsall and J. Duncan, Complete normed algebras, Springer-Verlag, New York Heidelberg Berlin (1973). MR 54:11013

[B1] J.W. Bunce, The joint spectrum of commuting nonnormal operators, Proc. Amer. Math. Soc. 29 (1971), 449-505. MR 44:832

[B2] J.W. Bunce, Models for n-tuples of noncommuting operators, J.Funct.Anal. 57 (1984), 21-30. MR 85k:47019

[C] L.A. Coburn, The $C^{*}$-algebra generated by an isometry, Bull.AMS 73 (1967), 722-726. MR 35:4760

[Cu] J. Cuntz, Simple $C^{*}$-algebras generated by isometries, Commun.Math.Phys. 57 (1977), 173-185. MR 57:7189

[D] J. Dixmier, $C^{*}$-algebras, North-Holland, Amsterdam (1977). MR 56:16388

[E] D.E. Evans, On $O_{n}$, Publ.Res.Int.Math.Sci. 16 (1980), 915-927. MR 82g:46099

[HW] W. Hurewicz and H. Wallman, Dimension Theory, Princeton University Press (1941). MR 3:312b

[PS] W.L. Paschke and N. Salinas,, Matrix algebras over $\mathcal{O}_{n}$, Michigan Math.J. 26 (1979), 3-12. MR 81a:46075

[P1] V.I. Paulsen, Every completely polynomially bounded operator is similar to a contraction, J.Funct.Anal. 55 (1984), 1-17. MR 86c:47021

[P2] V.I. Paulsen, Completely Bounded Maps and Dilations, Pitman Research Notes in Mathematics,Vol.146, New York, 1986. MR 88h:46111

[PP] M. Pimsner and S. Popa, The Ext-groups of some $C^{*}$-algebras considered by J. Cuntz, Rev.Roumaine Math.Pures Appl. 23 (1978), 1069-1076. MR 81j:46094

[Po1] G. Popescu, Models for infinite sequences of noncommuting operators, Acta.Sci. Math. 53 (1989), 355-368. MR 91b:47025

[Po2] G. Popescu, Isometric dilations for infinite sequences of noncommuting operators, Trans. Amer.Math.Soc. 316 (1989), 523-536. MR 90c:47006

[Po3] G. Popescu, Von Neumann inequality for $\left(B(H)^{n}\right)_{1}$, Math.Scand. 68 (1991), 292-304. MR 92k:47073

[Po4] G. Popescu, Multi-analytic operators on Fock spaces, Math.Ann. 303 (1995), 31-46. CMP 95:17

[Po5] G. Popescu, Functional calculus for noncommuting operators, Michigan Math.J. 42 (1995), 345-356. CMP 95:15

[S] W.F. Stinespring, Positive functions on $C^{*}$-algebras, Proc.Amer.Math.Soc. 6 (1955). MR 16:1033b

[SzF] B.Sz.-Nagy, C. Foias, Harmonic analysis on operators on Hilbert space, North-Holland, Amsterdam (1970). MR 43:947

[vN] J. von Neumann, Eine Spectraltheorie für allgemeine Operatoren eines unitären Raumes, Math.Nachr. 4 (1951), 258-281. MR 13:254a

Division of Mathematics, Computer Science and Statistics, The University of Texas at San Antonio, San Antonio, Texas 78249

E-mail address: gpopescu@ringer.cs.utsa.edu 